\title{
The Clinicopathologic Spectrum of IgG4-Related Disease
}

\author{
Ifeyinwa Emmanuela Obiorah, Alicia Henao Velasquez, Metin Özdemirli
}

Department of Pathology, Medstar Georgetown University Hospital, Washington, USA

Immunoglobulin G4-related disease is a fibroinflammatory systemic disease that is characterized by focal or diffuse organ infiltration by immunoglobulin G4-bearing plasma cells. Immunoglobulin G4-related disease may affect any organ, and a high index of suspicion is necessary for early detection to avoid irreversible fibrosis, organ dysfunction, and death. Tumor-forming lesions are common radiological features of immunoglobulin G4-related disease that need to be differentiated from malignancies. The diagnostic approach requires the integration of clinical, biochemical, and radiographic manifestations with classic histopathologic features, which remain crucial to diagnosis. The histology of immunoglobulin G4-related disease is determined by a dense lymphoplasmacytic infiltrate, storiform fibrosis, and obliterative phlebitis in the presence of increased immunoglobulin
G4-positve plasma cells. Although immunoglobulin G4-related disease forms a distinct, clinically independent disease category, many questions and problems remain unanswered, especially on its pathogenesis and the role of immunoglobulin G4. Advances in the understanding of immunoglobulin G4-related disease are likely to change the diagnostic approach in the future and create potential targets for therapeutic purposes. Here we describe the concept of immunoglobulin G4-related disease and the most recent knowledge in the clinico-pathological characteristics on this emerging disease. This study can guide clinicians in early diagnosis and prevent unnecessary surgical resections.

Keywords: Autoimmune, diagnosis, IgG4, IgG4-related disease, pathology, treatment
Immunoglobulin G4 (IgG4)-related disease (IgG4-RD) is a chronic immune-mediated disease that can affect any organ in the body. IgG4-RD was first described by Sarles et al. (1) in 1961 in a case of pancreatitis with hypergammaglobulinemia. In 1967, Comings and coworkers (2) reported a case of familial multifocal fibrosclerosis, which presented with features of retroperitoneal fibrosis, mediastinal fibrosis, sclerosing cholangitis, Riedel's thyroiditis, and pseudotumor of the orbit; these symptoms were all thought to be different manifestations of one disorder. In 2003, the extrapancreatic manifestations of IgG4-RD were first described by Kamisawa et al. (3) among a population of patients with autoimmune pancreatitis; this result suggested that multifocal fibrosclerosis in these patients were part of the same clinicopathologic spectrum. IgG4-RD tends to affect middle-aged to elderly individuals with a predilection for the male sex $(4,5)$. However, studies demonstrate a wide age range that includes pediatric patients (6). The pathogenesis of IgG4-RD remains largely unknown, and the exact role of the IgG4 molecule has not been identified. Data support that the inflammatory and fibrotic processes that drive IgG4-RD are initiated by a combination of T-helper type 2 (Th2) cells and regulatory $\mathrm{T}$ cells (Treg cells) (7). The disease entity can affect virtually any organ including the pancreas, liver, biliary system, salivary glands, lacrimal glands, retroperitoneum, lymph nodes, and kidney $(6,8)$, typically presenting as a mass or enlargement of one or more organ, which may mimic a malignancy (8). The diagnosis of IgG4-RD is vital to prevent unwarranted surgical resections, and it usually involves a combination of clinical, radiological, serological, and histopathological findings. Although most patients with IgG4-RD have an elevated serum IgG4 concentration, at least one-third of patients have a normal serum IgG4 concentration. Therefore, an elevated serum IgG4 is neither sensitive nor specific for the diagnosis and has a general role as a screening tool (5). The mainstay of diagnosis, regardless of the organ involved, is the identification of histopathologic features, which include dense lymphoplasmacytic inflammation, storiform fibrosis, obliterative phlebitis, and demonstration of dominance of IgG4-positive plasma cells (9). IgG4-RD can be challenging to diagnose when a less frequently affected organ is involved, and a high index of clinical suspicion is needed. This paper describes the potential pathogenesis and clinical, radiologic, and histopathological features of IgG4-RD and review responses related to therapy.

\section{PATHOPHYSIOLOGY OF IMMUNOGLOBULIN G4- RELATED DISEASE}

IgG4 is the least abundant in the IgG subclass and accounts for $<5 \%$ of the total IgG in healthy individuals (10). The overabundance of IgG4 antibodies may be due to the antibodies behaving as tissuedestructive immunoglobulins or excess IgG4 may simply be a

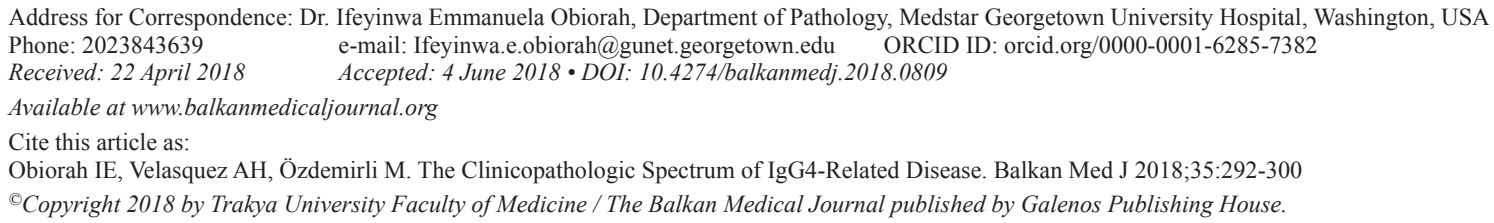


response to an unknown primary inflammatory stimulus (6). IgG4 molecule is not believed to be a driver of pathogenesis because it is involved in a continuous process of half molecular exchange, which is also known as Fab-arm exchange. This process involves the dissociation of the heavy chain dimers of an IgG4 molecule, and each half-molecule is associated with another different hemiIgG4 protein $(5,10)$. Most secreted IgG4 molecules behave as monovalent, non-cross-linking antibodies and cannot form large immune complexes or directly fix complements. Thus, IgG4 molecules are generally thought to be non-inflammatory, and they act to dampen rather than to incite or accelerate chronic immune activation. This function correlates with the well-known association of IgG4 with immunoglobulin E (IgE)-mediated allergy, where IgG4 antibodies induced by allergy treatments induce tolerance and protect allergic subjects from anaphylactic reactions by competing with allergen-specific IgE (11).

The exact mechanism of IgG4-RD remains unknown. One proposed mechanism is linked to Th2 cells, which are involved in the regulation of IgG4 production. Th2 cells secrete interleukins (IL-4, IL-5, and IL-13), which activate B cells to switch to IgG4 and IgE (5). Mattoo et al. (12) demonstrated that circulating Th2 memory cells accumulate among patients with IgG4-RD who have preexisting histories of atopy but not among those without atopic disease. This finding is unsurprising given that eosinophilia and increased serum IgE, which occur in $40 \%$ of patients with IgG4$\mathrm{RD}$, are mediated by Th2 cytokines (13). However, the precise mechanism of Th2-mediated IgG4-RD needs further clarification. Tregs may play a role in IgG4-RD, which is suggested by showing that Foxp3 messenger RNA, a transcription factor specific for CD4+ CD25+ Tregs, is significantly increased in IgG4-related pancreato-cholangitis in comparison with primary sclerosing cholangitis and primary biliary cirrhosis (14). In addition, Tregmediated expression of IL-10 and transforming growth factor- $\beta$ are overexpressed in IgG4-RD. IL-10 is implicated in the B cellinduced production of IgG4 (15), and transforming growth factor- $\beta$ is a fibrogenic cytokine that may be involved in the promotion of fibrosis in IgG4-RD. Another potential mechanism is the induction of CD4-positive T cells, which stimulate immune cells such as macrophages, myofibroblasts, and fibroblasts to drive fibrosis; this condition is presumably sustained by antigen-presenting B cells (5). This result may lead to the substantial improvement in IgG4RD following treatment with anti-CD20 therapy. Further evaluation of the immune-mediated pathway of IgG4-RD is necessary to generate potential targets that can result in the elimination of IgG4mediated inflammation and fibrosis.

\section{CLINICAL MANIFESTATIONS}

The clinical presentations of IgG4-RD are widely variable and can manifest in one or more organs synchronously or metachronously. IgG4-RD usually presents as a subacute or chronic process, and it can range from mild localized symptoms to major tissue damage and subsequent organ failure. Most patients are not constitutionally ill, so fever, malaise, night sweats, or weight loss are unusual (16). Tumorous lesions and allergic disease are common findings observed in IgG4-RD (6). One-third of patients with IgG4-RD have atopic disease, peripheral blood eosinophilia, and elevated IgE levels $(17,18)$. Nevertheless, a subset of nonatopic patients have peripheral blood eosinophilia and elevated IgE, suggesting that these conditions may be related to IgG4-RD itself rather than atopy. The most commonly reported manifestations are those related to the mass effect of pseudotumors in the affected organs or adjacent surrounding structures (19).

\section{Imaging Features}

Imaging is usually the first diagnostic tool to identify the tumorous swellings that occur in patients with IgG4-RD. Computed tomography (CT) may delineate a focal or diffuse pseudotumoral swelling of organs and identify the extent of multiorgan involvement. However, multiorgan involvement may be better assessed by $18 \mathrm{~F}$-fluorodeoxyglucose positron emission tomography/CT (18F-FDG PET/CT) (20,21). Imaging with 18F-FDG PET/CT enables delineation of the inflammation sites by identifying hypermetabolic activity to evaluate the extent of the disease, guide biopsy decision, and monitor response to treatment (22). Magnetic resonance imaging (MRI) of affected organs generates a low signal on T2-weighted imaging, with homogeneous enhancement reflecting increased cellularity and fibrosis (23). Imaging in patients with IgG4-RD typically reveals nonspecific findings, which are insufficient to differentiate the disorder from neoplastic conditions that present with mass-forming lesions. The exception to the rule is autoimmune pancreatitis, which shows classic CT or MRI findings of a diffusely enlarged sausage-shaped pancreas with a halo of edematous tissue and is pathognomonic for the disease entity (24). The major organ radiographic findings of IgG4-RD are summarized in Table 1.

\section{ORGAN MANIFESTATION}

\section{Pancreas}

The pancreas was the first organ associated with increased serum levels of IgG4 $(3,25)$. Two subtypes of autoimmune pancreatitis (AIP) have been described. Type 1 AIP is associated with IgG4$\mathrm{RD}$ and characterized by the classic histopathological findings of lymphoplasmacytic sclerosing pancreatitis (26). Type 2 AIP is not related to IgG4-RD and is recognized based on the identification of neutrophilic infiltrate into the pancreatic duct epithelium (27). The most frequent clinical manifestation of AIP is painless obstructive jaundice, which can mimic pancreatic cancer in the presence of pancreatic enlargement or mass lesion (Figure 1) (28). Endoscopic ultrasonography-guided fine needle aspiration can be attempted to exclude pancreatic cancer and should be performed before any empirical trial of steroids is undertaken. Less common clinical presentations include focal pancreatic mass, diffuse pancreatic enlargement, and pancreatic duct stricture (29). A minority of patients can present with severe abdominal pain with increased lipase levels, hence mimicking acute pancreatitis, or may present with features of chronic pancreatitis (6). In addition, some patients present with acute glucose intolerance, and treatment with corticosteroid improves glycemic control in a subset of patients (30). A characteristic feature of AIP, which belongs to the spectrum of IgG4-RD, is the involvement of other organs including bile 
duct stricture, renal involvement, retroperitoneal fibrosis, orbital pseudotumor, and diffuse lymphadenopathy $(28,29)$. These symptoms can serve as important supportive diagnostic clues. Although radiologic features of AIP are usually characteristic and may not warrant histologic diagnosis, the finding of a normal pancreas on imaging does not preclude AIP and requires further investigation.

\section{Hepatobiliary System}

IgG4-related sclerosing cholangitis is commonly associated with type 1 AIP. Solid mass lesions in hilar and perihilar ducts show close resemblance to primary sclerosing cholangitis and cholangiocarcinoma (31). Given that serum IgG4 concentrations nor cholangiographic or cholangioscopic features do not differentiate these disorders clearly (32-34), endoscopic biopsy is often required to differentiate IgG4-related sclerosing cholangitis from both primary sclerosing cholangitis and hilar cholangiocarcinoma. Histopathology is crucial for differential diagnosis. IgG4-related

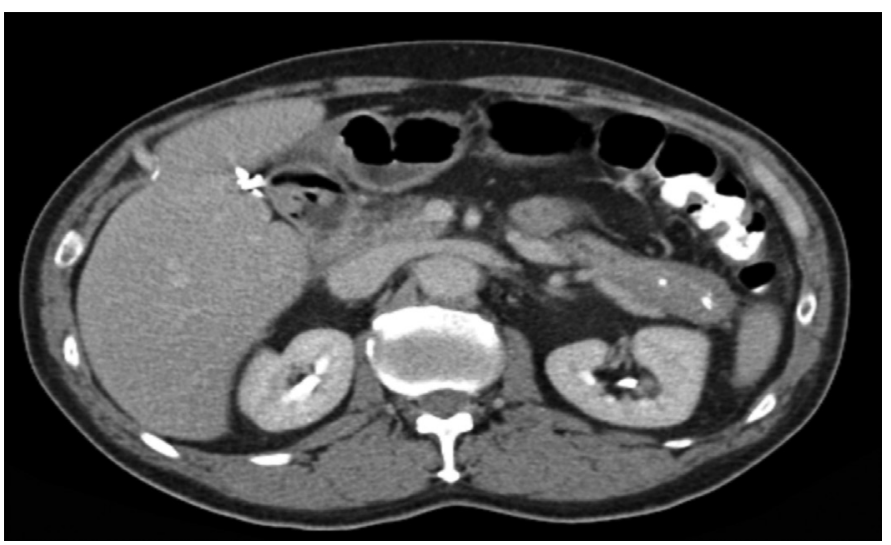

FIG. 1. Computed tomography scan of the pancreas. The image demonstrates a hypoattenuating pancreatic mass. hepatopathy has been described in patients with type 1 AIP and autoimmune hepatitis, or manifest as pseudotumors in the liver parenchyma (35).

\section{Salivary Glands}

Mikulicz's disease, which consists of symmetrical swelling of the lacrimal and salivary glands, is now recognized as IgG4-related dacryoadenitis and IgG4-related sialoadenitis (IgG4-RS) (6). Bilateral submandibular gland involvement is the most frequent presentation of IgG4-RS. However, the parotid, sublingual, and labial salivary glands may also be affected (36). IgG4-RS was previously considered to be a subset of Sjögren's syndrome (SS). Despite their similarities in organ involvement, IgG4-RS and SS are quite different conditions and need to be differentiated from each other. A comparison between IgG4-RS and SS reveals the following $(8,37)$ : in contrast to IgG4-RS, the enlargement of the parotid gland is predominant in SS. Patients with IgG4-RS have less symptoms of xerophthalmia, xerostomia, or arthralgia than patients with SS. Patients with IgG4-RS have coexisting AIP, interstitial nephritis, allergic rhinitis, and/or bronchial asthma, but anti-SS-A and anti-SS-B antibodies, rheumatoid factor, and antinuclear antibody are negative in most patients. Elevated serum IgG4 and marked infiltration with IgG4-positive plasma cells in IgG4-RS marks the most important difference between IgG4RS and SS. Finally, glucocorticoid therapy is highly effective in patients with IgG4-RD but has limited effect in patients with SS. Given that the radiographic findings of IgG4-RS are nonspecific, differentiation of multiglandular and localized involvement from malignant lymphoma for multiglandular disease and salivary gland carcinoma for localized disease is challenging.

\section{Orbits}

IgG4-RD can cause orbital inflammation that involves the lacrimal glands, extraocular muscles, orbital soft tissues, sclera, nasolacrimal

TABLE 1. Radiological features of immunoglobulin G4-related disease in major organs

\begin{tabular}{|c|c|}
\hline Organ & Imaging findings \\
\hline Pancreas $(28,29)$ & $\begin{array}{l}\text { Pancreatic enlargement with "sausage-shaped" appearance and a low-attenuating capsule-like "rim" around the enlarged } \\
\text { pancreas and pancreatic duct stricture }\end{array}$ \\
\hline Hepatobiliary system (35) & $\begin{array}{l}\text { Biliary stricture and dilatation, extrahepatic biliary stenosis, mass-forming lesion, biliary wall thickening, smooth inner and } \\
\text { outer margins, and/or a homogenous echo appearance of the internal bile duct wall }\end{array}$ \\
\hline Salivary gland $(40,80)$ & $\begin{array}{l}\text { Glandular enlargement, mass-forming lesions, multiple hypoechoic lesions, and homogenous enhancement of isointense/ } \\
\text { hypointense T2-weighed magnetic resonance imaging lesions }\end{array}$ \\
\hline Orbits $(38,41)$ & $\begin{array}{l}\text { Lacrimal gland enlargement, enlargement of the trigeminal nerve, or extraocular muscle and masses or hypertrophic lesions in } \\
\text { the ophthalmic tissues }\end{array}$ \\
\hline Ear, nose, and throat (43) & Swelling of mucosal surfaces, T2 iso/hypointense soft tissue thickening \\
\hline Thyroid gland $(50,51)$ & Homogenous thyroid gland enlargement with hypodense tissue, hypoechoicity on ultrasound \\
\hline Kidney (52) & $\begin{array}{l}\text { Low-density parenchymal lesions including mass-like lesions protruding beyond the surface of the kidney, rim-like lesion of the } \\
\text { kidney }\end{array}$ \\
\hline Retroperitoneum (56) & $\begin{array}{l}\text { Thick rim of homogenous retroperitoneal soft-tissue around the abdominal aorta, iliac artery, inferior vena cava, kidneys, and } \\
\text { urinary duct or psoas major, occurring symmetrically or asymmetrically }\end{array}$ \\
\hline Lungs $(60)$ & $\begin{array}{l}\text { Solid nodular densities; round-shaped ground-glass opacities; thickening of the bronchovascular bundles and interlobular septa; } \\
\text { alveolar interstitial type with bronchiectasis resembling interstitial lung disease and pleural thickening }\end{array}$ \\
\hline Gastrointestinal system (63) & Diffusely thickened mucosa, submucosal mass-like lesions \\
\hline Central nervous system (66) & Linear dural thickening or a bulging mass; sellar enlargement and thickening of the pituitary stalk \\
\hline
\end{tabular}


duct, and trigeminal nerve; it is sometimes accompanied with bone destruction and development of a saddle nose deformity (38). Unlike systemic IgG4-RD, patients with IgG4-related orbital disease (IgG4-ROD) affect both male and female sexes equally (39). Patients with IgG4-ROD often present with painless bilateral orbitopathy. The clinical symptoms are indolent and often occur as a result of regional mass effect or diffuse infiltration of involved tissues, including painless proptosis, diplopia, and impaired eye movements (40). IgG4-ROD is usually bilateral than unilateral at presentation. By contrast, patients with "orbital pseudotumor" or "idiopathic orbital inflammation," which is not related to IgG4, tend to be unilateral and usually present with an acute onset of pain, erythema, proptosis, and restriction of ocular motility (41). Nonetheless, a considerable number of cases may be IgG4-related ophthalmic disease. Other considerations in the differential diagnosis of patients with IgG4-ROD include SS, granulomatosis with polyangiitis, sarcoidosis, lymphoma, infection, Graves' thyrotoxicosis, and cancer (38).

\section{Ear, Nose, and Throat}

Sinonasal manifestations of IgG4-RD, although rare, have been reported and are now a recognized entity with two patterns of presentation (40). Patients may present as a destructive locally invasive mass, which can manifest as nonspecific symptoms such as nasal obstruction, rhinorrhea, epistaxis, facial pain, and anosmia (42-44). Frequent sites of involvement include the maxillary sinus, nasal cavity, and septum, whereas the sphenoid sinus and anterior ethmoid air cells are less commonly affected. Sinonasal IgG4$\mathrm{RD}$ has a predilection for bone destruction, thereby mimicking sinonasal lymphoma (45). The second pattern of sinonasal IgG4$\mathrm{RD}$ is diffuse infiltration of the sinonasal mucosa with an IgG4positive plasmacytic infiltrate. This manifestation closely resembles "idiopathic chronic rhinosinusitis," which also demonstrates IgG4 plasma cells in the mucosa, thereby making differentiation between the two disorders very difficult (46). As previously noted, allergic features occur in a substantial subset of patients with IgG4$\mathrm{RD}$, so an allergic pathophysiology may be responsible for the prevalence of IgG4-related chronic rhinosinusitis in these patients (47). Otologic involvement with IgG4-RD can manifest as otitis media with effusions, eosinophilic otitis media, and sensorineural hearing loss. Takagi et al. (48) reported five cases that responded to glucocorticoid therapy. Although the patients had biopsy-proven IgG4-RD at other sites, none of the otologic cases were confirmed by biopsy.

\section{Thyroid}

Thyroid gland IgG4-RD includes Reidel's thyroiditis and fibrosing Hashimoto's thyroiditis. Reidel's thyroiditis is a chronic fibrosclerotic disorder that was first described by Reidel (49) in 1896; it manifests as a hard infiltrative lesion in the thyroid gland. Dahlgren and coauthors (50) demonstrated increased IgG4-positive plasma cells and the morphologic features of IgG4-RD in patients with Reidel's thyroiditis. The fibrosing variant of Hashimoto's thyroiditis is now considered part of the clinical spectrum of IgG4$\mathrm{RD}$, and it is characterized by dense bands of fibrosis and marked infiltrations of IgG4-positive plasma cells (36). Compared with
Riedel's thyroiditis, extra-thyroidal fibrosis is absent in fibrosing Hashimoto's thyroiditis. In addition, patients with IgG4-related Hashimoto's thyroiditis tend to be young men with shorter duration of the disease compared with those with the non-IgG4-associated subtype of Hashimoto's disease (51).

\section{Kidney}

IgG4-related kidney disease (IgG4-RKD) usually becomes clinically obvious when there is renal dysfunction or discovered incidentally as multiple low-density lesions on imaging (52). Common findings of IgG4-RKD include tubulointerstitial nephritis (TIN) and membranous glomerulonephritis (MGN). TIN has the typical histologic pattern of IgG4-RD with tubular destruction and atrophy. The presence of IgG4-positive plasma cells is sensitive but not specific for IgG4-TIN. IgG4-bearing plasma cells have been described in necrotizing glomerulonephritis, diabetic nephropathy, lupus nephritis, MGN, and idiopathic TIN (52). Features that can help differentiate IgG4-TIN from other entities include storiform pattern of fibrosis, well-demarcated margins of involvement, and lymphoplasmacytic cell infiltration into and beyond the renal capsule, which is reflected occasionally as a rim-like lesion of the kidney noted on CT (53). MGN is part of the IgG4-RD spectrum and needs to be differentiated from primary MGN. A primary marker of primary MGN, anti-M-type phospholipase A2 receptor antibody, is typically negative in IgG4-MGN (54). In contrast to primary MGN, granular $\mathrm{C} 1 \mathrm{q}$ deposits are sometimes prominent, and concurrent IgG4-TIN can occur in IgG4-MGN. Other glomerular diseases involved in IgG4-RKD include IgA nephropathy, IgA vasculitis, and membranoproliferative glomerulonephritis (55).

\section{Retroperitoneum}

IgG4-related retroperitoneal fibrosis (IgG4-RRPF) is characterized by chronic inflammatory and infiltration of fibrotic tissue in the periaortic or periiliac retroperitoneum that encases adjacent structures (56). Different locations of involvement result in varying clinical manifestations. Kidney and urinary tract damage causes hydronephrosis, backache, and acute renal failure. Sclerosing mesenteritis can result from compression on the inferior vena cava, which may cause clinical symptoms of abdominal pain, diarrhea, intestinal obstruction, and ischemia (56). IgG4-related periaortitis and periarteritis are the most common lesions affecting various medium- to large-sized arteries, including the abdominal aorta, iliac artery, renal artery, and mesenteric artery (57). Inflammatory aneurysms are common complications of IgG4-related aortitis, which may present with a pulsatile mass, low-grade fever, back pain, or aneurysm rupture. IgG4-RRPF and idiopathic retroperitoneal fibrosis have similar clinical and imaging features. The two can be distinguished by the presence of classic IgG4-related histopathologic findings and elevated serum IgG4 concentration (58).

\section{Lymph Nodes}

IgG4-related lymphadenopathy (IgG4-RL) may be seen incidentally in excisions made for extranodal disease or detected in patients with symptomatic systemic IgG4-RD (59). IgG4-RL can be generalized or localized and may be the only manifestation 
of IgG4-RD. The lymph nodes are usually painless and $1-5 \mathrm{~cm}$ in size. The frequent sites of involvement include the cervical, supraclavicular, mediastinal, pulmonary-hilar, abdominal, axillary, or inguinal groups. Diagnosis of IgG4-RD in lymph nodes can be difficult because fibrosis is infrequent and typically seen in pseudotumor-like lymph nodes (5). Differential diagnosis of IgG4-RL should include infections, lymphoma, carcinoma, and Castleman's disease.

\section{Lungs}

Clinical symptoms observed in patients with IgG4-related lung disease are nonspecific and include cough, chest pain, and dyspnea (60). Low-grade fever and weight loss may occur but are often uncommon. Pulmonary parenchymal involvement can manifest as a pseudotumor on imaging and account for some cases previously diagnosed as inflammatory pseudotumor (plasma cell granuloma) in the lungs (61). Interstitial lung disease is another tissue manifestation of IgG4-related lung disease, and the disease may closely resemble nonspecific interstitial pneumonia, organizing pneumonia, or lymphoid interstitial pneumonia (62). Given that these patterns are not specific, histopathologic examination remains critical to diagnosis.

\section{Gastrointestinal Disease}

IgG4-related gastrointestinal disease is a newly evolving disorder in the IgG4-RD family. The clinical manifestations of IgG4-related gastrointestinal disease can either present as a marked thickening of the gastrointestinal wall consisting of marked fibrosis with increased infiltration of IgG4-bearing plasma cells or as an IgG4-
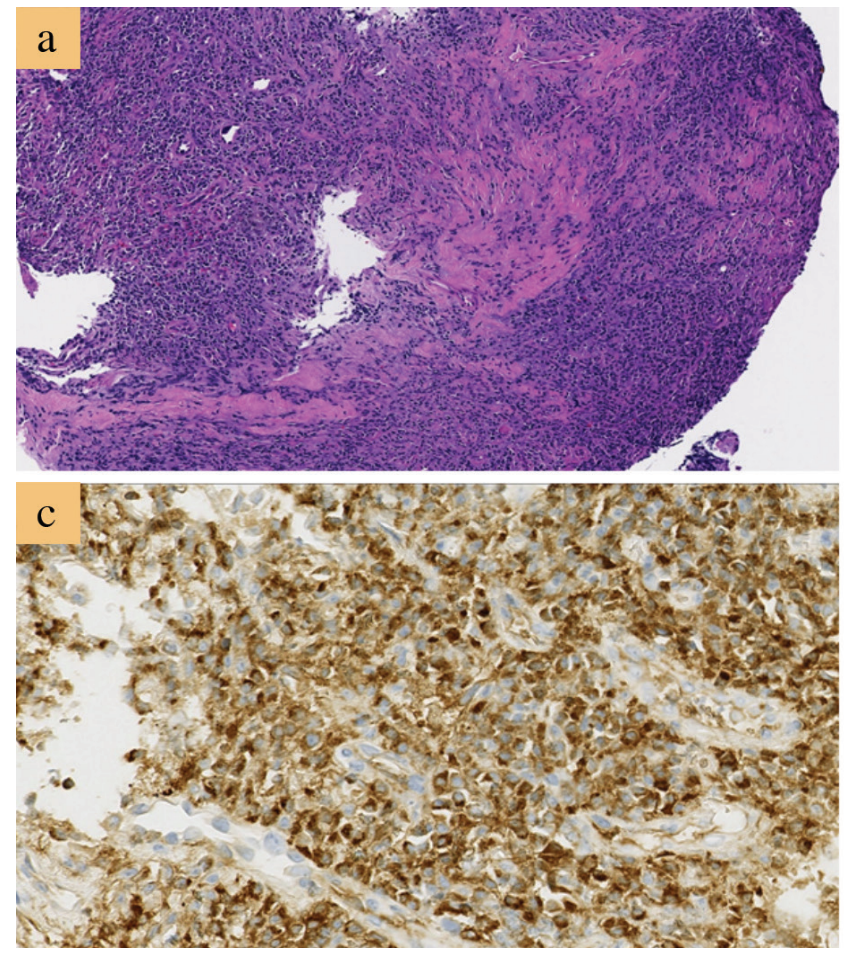

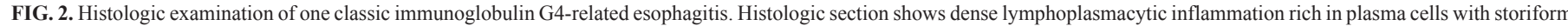
fibrosis and obliterative phlebitis (hematoxylin and eosin) stain, a) $40^{\times}$; b) $400^{\times}$. Majority of the plasma cells are positive for IgG, c) and immunoglobulin G4, d) (immunohistochemistry, $400^{\times}$, each.). Reprinted from Obiorah et al. (64). Reprinted with permission of Oxford University Press. related pseudotumor (63). IgG4-related esophagitis is a rare entity. The most frequent symptom is dysphagia, and esophageal stricture is the most common finding via endoscopy (64). Patients with idiopathic chronic esophagitis can be difficult to differentiate from IgG4-related esophagitis because they have similar manifestations such as strictures (64). Thus, histopathologic examination is essential for the diagnosis of IgG4-related esophagitis (Figure 2). Although uncommon, IgG4-related esophagitis should be considered in any case of unexplained stricture in the absence of malignancy. IgG4-related gastritis can show diffusely thickened gastric mucosa on endoscopy or present as submucosal pseudotumors. Esophageal strictures due to IgG4-RD (Figure 3a) have been shown to be responsive to corticosteroid therapy (Figure 3b). Rarely, IgG4-related gastritis can present as refractory gastric ulcers that are unresponsive to Helicobacter pylori treatment (65). Polypoid or mass-like lesions are common findings in IgG-RD involving the major duodenal papilla and colon (63). Malignancy should be ruled out, especially in the absence of another organ's involvement, to avoid unnecessary resection.

\section{Neurological Involvement}

IgG4-RD has been infrequently reported in the central nervous system, and it has a specific propensity for the involvement of the meninges and cranial nerves. IgG4-related hypertrophic pachymeningitis can cause localized or diffuse thickening of the dura mater (66). Frequent symptoms at presentation include headache, cranial nerve palsies, vision disturbances, motor weakness, limb numbness, sensorineural hearing loss, neck stiffness, and seizures. Involvement of cranial nerves usually results from adjacent mass-
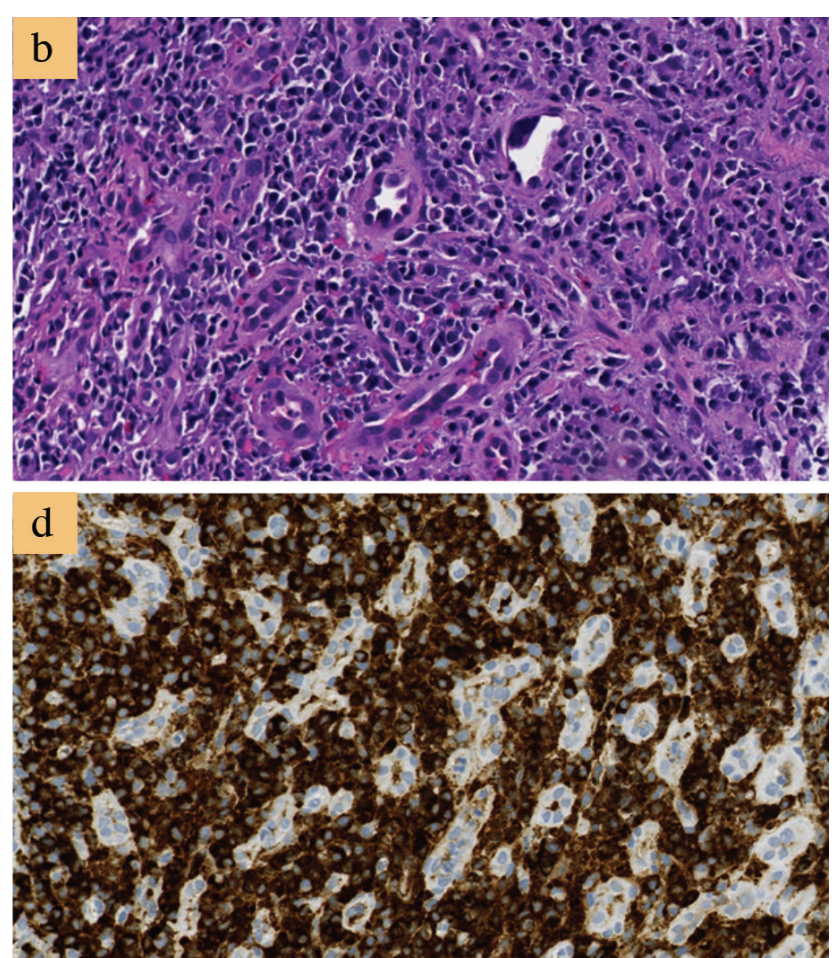
like lesions (67). Cerebrospinal fluid analysis is often nonspecific and cannot effectively differentiate IgG4-related pachymeningitis from other forms of inflammation. Histologic examination of the meninges is the gold standard for the diagnosis. Clinical manifestation of IGg4-RD involving the pituitary gland depends on the size and location of the lesion within the gland. Therefore, IgG4-related hypophysitis can result in hormone deficiencies from both the anterior and posterior pituitary (68).

\section{Other Organs}

Skin can be involved in IgG4-RD. Two main cutaneous lesions are erythematous plaques and subcutaneous nodules. Other lesions such as brown hyperpigmented papules in patients with dark pigmented skin occur less commonly (69). Typical sites affected include the skin of the head and neck region, and the less affected regions are the trunk and limbs. Involvement of the prostate has also been reported, usually as a presumptive diagnosis based on the presence of IgG4-RD in other organs and resolution of an apparent benign prostatic hypertrophy following glucocorticoid treatment (66). However, biopsy-proven mass-forming IgG4-related prostatitis has also been reported (66). IgG4-related mastitis has been described in five cases and tends to present as painless mass lesions $(66,70)$. Testicular involvement by IgG4-RD can occur as a paratesticular pseudotumor or epididymo-orchitis (71).

\section{DIAGNOSIS}

\section{Laboratory Parameters}

The diagnosis of IgG4-RD depends on the combination of clinical, radiological, pathological, and laboratory modalities including serology. Although quantification of the serum IgG4 concentration is included in all IgG4-RD diagnostic guidelines, approximately one-third of patients with biopsy-proven IgG4-RD have normal serum IgG4 concentrations; thus, serum IgG4 concentration is not required for the diagnosis of IgG4-RD (6). Besides, increased serum IgG4 levels have been observed in patients with a variety of other diseases including primary sclerosing cholangitis (32), making it an insufficient single diagnostic tool. Increased serum IgG4 (typically $>135 \mathrm{mg} / \mathrm{dL}$ ) identifies patients with an active form of the disease, which is correlated with increased concentrations of inflammatory serum biomarkers and hypocomplementemia, increased number of organs affected by the disease, and extensive organ involvement (72). These patients appear to have a shorter time to disease relapse than patients with IgG4-RD with normal serum IgG4 at the time of diagnosis. Serum IgG4 levels usually decrease with glucocorticoid therapy, but they are not diseasespecific (73). Some patients with IgG4-RD may remain in remission despite having persistent elevated serum IgG4 levels (6). Elevated circulating plasmablasts have been observed in patients with IgG4-RD (74). The increased levels of plasmablasts correlate with disease activity even in the presence of normal serum IgG4 levels. Increased circulating plasmablasts appear to be superior to serum IgG4, but their use as biomarkers of disease activity is still poorly characterized; further studies are needed before their broad use can be endorsed. In certain cases of IgG4-RD, especially those involving the kidney, complement levels are a useful indicator of disease activity. Hypocomplementemia has been observed at the time of relapse in patients with IgG4-related TIN (53).

\section{Histology}

The current diagnostic criteria from the 2012 consensus statement (9) for the histopathologic features of IgG4-RD include a triad of dense lymphoplasmacytic infiltrate, fibrosis arranged at least focally in a storiform manner (Figure 4a), and obliterative phlebitis in the background of increased numbers of IgG4-positive plasma cells (Figure 4b). In most cases, a confident pathological diagnosis of IgG4-RD can be made in the presence of two of the three major histological features. Other histopathologic features, though neither sensitive nor specific, that can be found in IgG4-RD include phlebitis without obliteration of the lumen and elevated numbers of eosinophils. In addition, the presence of $>10$ IgG4+ plasma cells on biopsy material has been proposed as one element of a comprehensive diagnostic panel. However, the appropriate cutoff point may vary depending on the organ involved and based on the predominance of fibrosis at the time the diagnosis is made. For instance, cases of IgG4-RRPF will have extensive areas of dense fibrosis, which may make identification of IgG4positive plasma cells challenging (58). Hence, the $\mathrm{IgG} 4 / \mathrm{IgG}$ ratio is a powerful tool in establishing a diagnosis of IgG4-RD. Studies $(24,66)$ have suggested an IgG4/IgG plasma cell ratio of $>40 \%$ as a
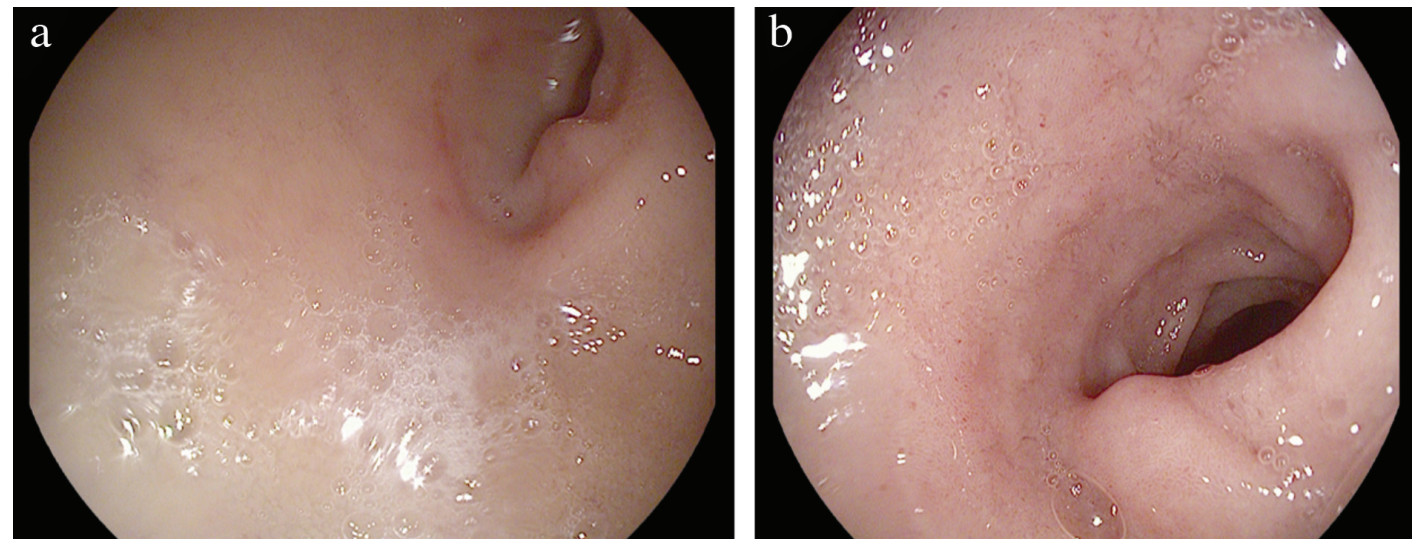

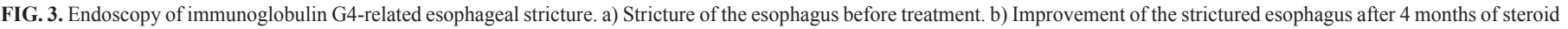
therapy. Reprinted from Obiorah et al. (64). Reprinted with permission of Oxford University Press. 
comprehensive cutoff value in any organ, and this ratio is generally adopted as a histological diagnostic criterion for IgG4-RD. Certain disorders exhibit elevated levels of IgG4-bearing plasma cells in tissue specimens such as primary sclerosing cholangitis, antineutrophil cytoplasmic antibody-associated vasculitis, rheumatoid arthritis, inflammatory bowel disease, rhinosinusitis, RosaiDorfman disease, autoimmune atrophic gastritis, and multicentric Castleman's disease (9). However, these conditions all lack the classic characteristic histopathological features of IgG4-RD.

\section{Clinical Diagnostic Criteria}

Although the histopathological findings can provide strong supportive evidence for the diagnosis of IgG4-RD, correlation with the clinical presentation and radiographic findings is often required to make a definitive diagnosis. Some organ-specific diagnostic criteria exist for certain organs such as IgG4-related sclerosing cholangitis (31), but confusion may present when IgG4-RD affects a rare organ for which diagnostic criteria are non-existent. No validated diagnostic criteria exist for IgG4-RD, but the diagnosis should be based on the following characteristics $(20,24)$ : (a) mass lesions in one or more organs; (b) lymphoplasmacytic infiltrate, fibrosis, and obliterative phlebitis with $>40 \%$ of IgG+ plasma cells being IgG4+ and $>10$ cells/high-power field of biopsy sample; and (c) serum IgG4 concentration $>135 \mathrm{mg} / \mathrm{dL}$. A definite diagnosis is considered when all three criteria (a-c) are met. The diagnosis of IgG4-RD is considered probable when (a) and (b) are met. When (a) and (c) are present, it is defined as possible diagnosis. Treatment is generally advocated in patients with either a definite and probable diagnosis especially in patients with an active disease.

\section{TREATMENT}

\section{Corticosteroid Therapy}

Most patients with IgG4-RD respond to corticosteroids, which remain the first line of treatment. A suggested treatment scheme based on the international consensus guidance statement $(20,75)$ on the management of IgG4-RD includes induction therapy with $40 \mathrm{mg}$ of prednisone daily with assessment for treatment response at 2-4 weeks using clinical, radiologic, and biochemical markers. Treatment should be tapered if response is good; otherwise, burnt out disease or an alternate diagnosis should be considered. Tapering of prednisone should be done over 3-6 months to $5 \mathrm{mg}$ daily (6). Maintenance therapy should be considered if the patient has multiorgan disease, significantly elevated serum IgG4, prior history of relapse, and system-specific markers. Maintenance treatment can include low dose of prednisone $(2.5-5 \mathrm{mg}$ /day for up to 3 years) or the use of a glucocorticoid sparing agent such as azathioprine $(20,75)$. The response to steroid therapy varies depending on the involved organs and the degree of fibrosis (6). AIP generally responds to glucocorticoids, and pancreatic function can improve following treatment $(27,29)$. By contrast, IgG4-RRPF is less amenable to corticosteroid treatment (56).

\section{Corticosteroid Sparing Agents}

Conventional corticosteroid sparing drugs, such as azathioprine, mycophenolate mofetil, and methotrexate, are widely used either to achieve additional immunosuppression or as steroid sparing agents (76). Both prospective and retrospective studies show that corticosteroids are initially effective for most patients, but they are often poorly tolerated, and disease relapses during or following tapering of corticosteroids are common (75). Although clinical improvement has been described in patients treated with these agents $(27,64)$, none has been tested in prospective, controlled studies. Hart et al. (77) retrospectively compared the results of treatment of patients with relapsing AIP with azathioprine, mycophenolate mofetil, and 6-mecaptopurine with corticosteroid therapy, and they found that the relapse-free survival did not significantly differ between the two groups. Therefore, further evidence for the efficacy of conventional steroid sparing agents in IgG4-RD is needed.

\section{Rituximab}

Rituximab, an anti-CD20 monoclonal antibody, induces B-cell depletion to result in a decrease in IgG4-positive plasma cells and serum IgG4 (77,78). In addition, IgG-positive plasmablasts rapidly decline following treatment with rituximab in patients with IgG4-
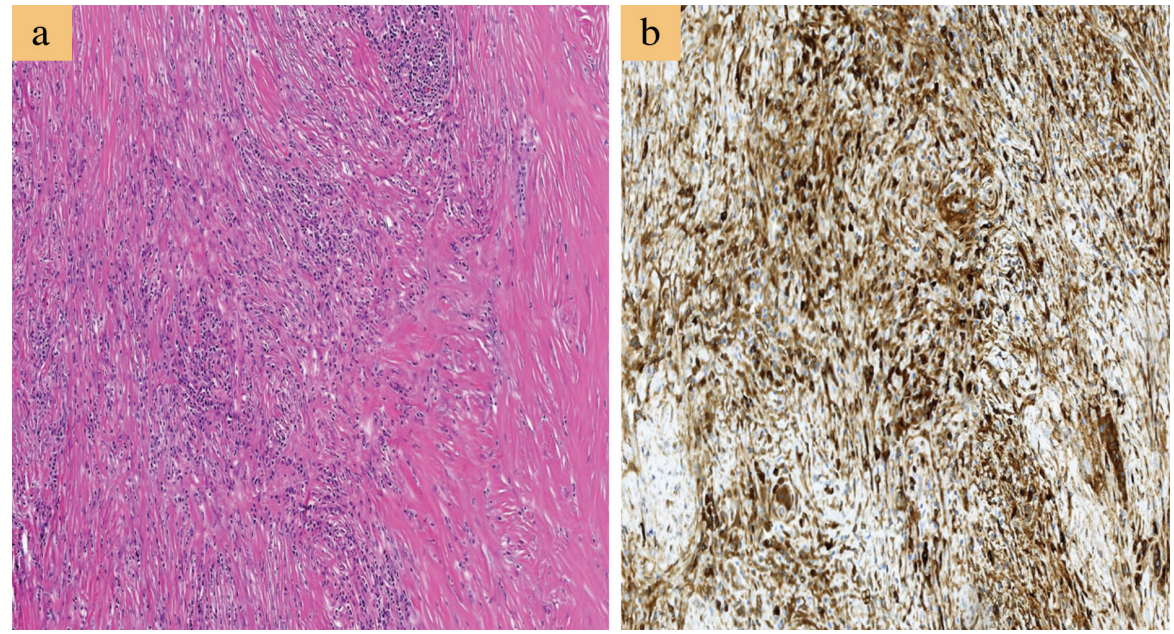

FIG. 4. a, b. Histologic examination of a case of immunoglobulin G4-related pancreatitis. a) Histologic section shows dense lymphoplasmacytic inflammation rich in plasma cells with storiform fibrosis (hematoxylin and eosin stain, $100^{\times}$). Majority of the plasma cells are positive for immunoglobulin G4 (D; immunohistochemistry, $200^{\times}$). 
RD (74). Reports from case series indicate that B-cell depletion with rituximab might be an effective therapy for treating IgG4$\mathrm{RD}$, but this type of treatment was used in patients who did not respond to corticosteroids, conventional steroid-sparing agents, or both $(77,78)$. Carruthers et al. (79) evaluated the efficacy of rituximab in an open-label pilot trial, in which $83 \%$ of 30 patients received rituximab monotherapy. Disease responses were observed in $97 \%$ of patients, and the primary outcome achieved by 23 of 30 patients revealed that $47 \%$ were in complete remission at 6 months, which was sustained at 12 months in $40 \%$ of the patients. Although rituximab shows tremendous promise in the treatment of IgG4$\mathrm{RD}$, further evaluation is required in the setting of large controlled clinical trials (80).

IgG4-RD is a widely recognized systemic disorder that exhibits pathological features, which remain consistent in any affected organ. Substantial progress has been achieved in the diagnosis and management of IgG4-RD, but many questions remain unanswered in determining the pathogenesis that lies behind the disease, which may be the key to the development of an effective targeted therapy. The clinical manifestations of the disorder are usually nonspecific and vary from organ to organ; thus, detection in early stages can be challenging. Irreversible fibrosis and organ failure can develop if untreated, so clinicians in various specialties should be aware of the disease. The diagnostic approach is complex and requires a combination of clinical, laboratory, and radiological examinations with typical pathological findings. Corticosteroids remain the first line of therapy in active IgG4-RD, but adverse effects of the drug and disease recurrences on reduction or termination of therapy highlight the need for favorable therapy. Rituximab appears to be a promising agent in the treatment of IgG4-RD, but its efficacy needs to be evaluated in large clinical controlled trials.

Conflict of Interest: No conflict of interest was declared by the authors.

Financial Disclosure: No financial disclosure was declared by the authors.

\section{REFERENCES}

1. Sarles H, Sarles JC, Muratore R, Guien C. Chronic inflammatory sclerosis of the pancreas--an autonomous pancreatic disease? Am J Dig Dis 1961;6:688-98.

2. Comings DE, Skubi KB, Van Eyes J, Motulsky AG. Familial multifocal fibrosclerosis: Findings suggesting that retroperitoneal fibrosis, mediastinal fibrosis, sclerosing cholangitis, riedel's thyroiditis, and pseudotumor of the orbit may be different manifestations of a single disease. Ann Intern Med 1967;66:884-92.

3. Kamisawa T, Funata N, Hayashi Y, Tsuruta K, Okamoto A, Amemiya K, et al. Close relationship between autoimmune pancreatitis and multifocal fibrosclerosis. Gut 2003;52:683-7.

4. Wallace ZS, Deshpande V, Mattoo H, Mahajan VS, Kulikova M, Pillai S, et al. IgG4Related Disease: clinical and laboratory features in 125 patients. Arthritis Rheumatol 2015;67:2466-75.

5. Kamisawa T, Zen Y, Pillai S, Stone JH. IgG4-related disease. Lancet 2015;385:1460-71.

6. Stone JH, Zen Y, Deshpande V. IgG4-Related Disease. N Eng J Med 2012;366:539-51.

7. Zen Y, Nakanuma Y. Pathogenesis of IgG4-related disease. Curr Opin Rheumatol 2011;23:114-8.

8. Okazaki K, Umehara H. Current Concept of IgG4-Related Disease. Curr Top Microbiol Immunol 2017;401:1-17.

9. Deshpande V, Zen Y, Chan JK, Yi EE, Sato Y, Yoshino T, et al. Consensus statement on the pathology of IgG4-related disease. Mod pathol 2012;25:1181-92.

10. Aalberse RC, Stapel SO, Schuurman J, Rispens T. Immunoglobulin G4: an odd antibody. Clin exp allerg 2009;39:469-77.
11. Durham SR, Emminger W, Kapp A, Colombo G, de Monchy JG, Rak S, et al. Longterm clinical efficacy in grass pollen-induced rhinoconjunctivitis after treatment with SQ-standardized grass allergy immunotherapy tablet. J Allergy Clin Immunol 2010;125:131-8

12. Mattoo H, Della-Torre E, Mahajan VS, Stone JH, Pillai S. Circulating Th2 memory cells in IgG4-related disease are restricted to a defined subset of subjects with atopy. Allergy 2014;69:399-402.

13. Kamisawa T, Anjiki H, Egawa N, Kubota N. Allergic manifestations in autoimmune pancreatitis. Eur J Gastroenterol Hepatol 2009;21:1136-9.

14. Zen Y, Fujii T, Harada K, Kawano M, Yamada K, Takahira M, et al. Th2 and regulatory immune reactions are increased in immunoglobin G4-related sclerosing pancreatitis and cholangitis. Hepatology 2007;45:1538-46.

15. Jeannin P, Lecoanet S, Delneste Y, Gauchat JF, Bonnefoy JY. IgE versus IgG4 production can be differentially regulated by IL-10. J Immunol 1998;160:3555-61.

16. Yamamoto M, Takahashi H, Shinomura Y. Mechanisms and assessment of IgG4related disease: lessons for the rheumatologist. Nat Rev Rheumatol 2013;10:148-59.

17. Della Torre E, Mattoo H, Mahajan VS, Carruthers M, Pillai S, Stone JH. Prevalence of atopy, eosinophilia, and IgE elevation in IgG4-related disease. Allergy 2014;69:269-72.

18. Chen H, Lin W, Wang Q, Wu Q, Wang L, Fei Y, et al. IgG4-related disease in a Chinese cohort: a prospective study. Scand J Rheumatol 2014;43:70-4.

19. Martinez-Valle F, Fernandez-Codina A, Pinal-Fernandez I, Orozco-Galvez O, Vilardell-Tarres M. IgG4-related disease: Evidence from six recent cohorts. Autoimmun Rev 2017; 16:168-72.

20. Haldar D, Cockwell P, Richter AG, Roberts KJ, Hirschfield GM. An overview of the diagnosis and management of immunoglobulin G4-related disease. CMAJ 2016;188:953-61.

21. Zhang J, Chen H, Ma Y, Xiao Y, Niu N, Lin W, et al. Characterizing IgG4-related disease with 18F-FDG PET/CT: a prospective cohort study. Eur J Nucl Med Mol Imag 2014;41:1624-34.

22. Vasaitis L. IgG4-related disease: A relatively new concept for clinicians. Eur J Int Med 2016;27:1-9.

23. Toyoda K, Oba H, Kutomi K, Furui S, Oohara A, Mori H, et al. MR imaging of IgG4-related disease in the head and neck and brain. AJNR Am J Neuroradiol 2012;33:2136-9.

24. Umehara H, Okazaki K, Masaki Y, Kawano M, Yamamoto M, Saeki T, et al. Comprehensive diagnostic criteria for IgG4-related disease (IgG4-RD), 2011. Mod Rheumatol 2012;22:21-30.

25. Hamano H, Kawa S, Horiuchi A, Unno H, Furuya N, Akamatsu T, et al. High serum IgG4 concentrations in patients with sclerosing pancreatitis. N Eng J Med 2001;344:732-8.

26. Sah RP, Chari ST, Pannala R, Sugumar A, Clain JE, Levy MJ, et al. Differences in clinical profile and relapse rate of type 1 versus type 2 autoimmune pancreatitis. Gastroenterol 2010;139:140-8.

27. Hart PA, Kamisawa T, Brugge WR, Chung JB, Culver EL, Czako L, et al. Longterm outcomes of autoimmune pancreatitis: a multicentre, international analysis. Gut 2013;62:1771-6.

28. Sugumar A, Chari ST. Autoimmune pancreatitis. J Gastroenterol Hepatol 2011;26:1368-73.

29. Majumder S, Takahashi N, Chari ST. Autoimmune Pancreatitis. Dig Dis Sci 2017;62:1762-9.

30. Kamisawa T, Egawa N, Inokuma S, Tsuruta K, Okamoto A, Kamata N, et al Pancreatic endocrine and exocrine function and salivary gland function in autoimmune pancreatitis before and after steroid therapy. Pancreas 2003;27:235-8.

31. Nakazawa T, Naitoh I, Hayashi K, Okumura F, Miyabe K, Yoshida M, et al Diagnostic criteria for IgG4-related sclerosing cholangitis based on cholangiographic classification. J Gastroenterol 2012;47:79-87.

32. Mendes FD, Jorgensen R, Keach J, Katzmann JA, Smyrk T, Donlinger J, et al. Elevated serum IgG4 concentration in patients with primary sclerosing cholangitis. Am J Gastroenterol 2006;101:2070-5.

33. Oseini AM, Chaiteerakij R, Shire AM, Ghazale A, Kaiya J, Moser CD, et al. Utility of serum immunoglobulin G4 in distinguishing immunoglobulin G4-associated cholangitis from cholangiocarcinoma. Hepatology 2011;54:940-8.

34. Kalaitzakis E, Levy M, Kamisawa T, Johnson GJ, Baron TH, Topazian MD, et al. Endoscopic retrograde cholangiography does not reliably distinguish IgG4-associated cholangitis from PSC or cholangiocarcinoma. Clin Gastroenterol Hepatol 2011;9:800-3. 
35. Culver EL, Chapman RW. IgG4-related hepatobiliary disease: an overview. Nat rev Gastroenterol Hepatol 2016;13:601-12.

36. Takano K, Yamamoto M, Takahashi H, Himi T. Recent advances in knowledge regarding the head and neck manifestations of IgG4-related disease. Auris Nasus Larynx 2017;44:7-17.

37. Umehara H, Okazaki K, Masaki Y, Kawano M, Yamamoto M, Saeki T, et al. A novel clinical entity, IgG4-related disease (IgG4RD): general concept and details. Mod Rheumatol 2012;22:1-14.

38. Wallace ZS, Deshpande V, Stone JH. Ophthalmic manifestations of IgG4-related disease: Single-center experience and literature review. Semin Arthritis Rheum 2014;43:806-17.

39. Andrew N, Kearney D, Selva D. IgG4-related orbital disease: a meta-analysis and review. Acta Ophthalmol 2013;91:694-700.

40. Thompson A, Whyte A. Imaging of IgG4-related disease of the head and neck. Clin Radiol 2018;73:106-20.

41. Goto H, Takahira M, Azumi A; Japanese Study Group for IgG4-Related Ophthalmic Disease. Diagnostic criteria for IgG4-related ophthalmic disease. Jpn J Ophthalmol 2015;59:1-7

42. Ishida M, Hotta M, Kushima R, Shibayama M, Shimizu T, Okabe H. Multiple IgG4related sclerosing lesions in the maxillary sinus, parotid gland and nasal septum. Pathol Int 2009;59:670-5.

43. Prabhu SM, Yadav V, Irodi A, Mani S, Varghese AM. IgG4-related disease with sinonasal involvement: A case series. Indian J Radiol Imaging 2014;24:117-20.

44. Cain RB, Colby TV, Balan V, Patel NP, Lal D. Perplexing lesions of the sinonasal cavity and skull base: IgG4-related and similar inflammatory diseases. Otolaryngol Head Neck Surg 2014;151:496-502.

45. Pace C, Ward S. A rare case of IgG4-related sclerosing disease of the maxillary sinus associated with bone destruction. J Oral Maxillofac Surg 2010;68:2591-3.

46. Moteki H, Yasuo M, Hamano H, Uehara T, Usami S. IgG4-related chronic rhinosinusitis: a new clinical entity of nasal disease. Acta Otolaryngol 2011;131:518-26.

47. Takano K, Abe A, Yajima R, Kakuki T, Jitsukawa S, Nomura K, et al. Clinical Evaluation of Sinonasal Lesions in Patients With Immunoglobulin G4-Related Disease. Ann Otol Rhinol Laryngol 2015;124:965-71.

48. Takagi D, Nakamaru Y, Fukuda S. Otologic Manifestations of Immunoglobulin G4Related Disease. Ann Otol Rhinol Laryngol 2014;123:420-4.

49. Riedel B. Die chronische, zur Bildung eisenharter Tumoren führende Entzündung der Schilddrüse. Verh Dtsch Ges Chir 1896;25:101-5.

50. Dahlgren M, Khosroshahi A, Nielsen GP, Deshpande V, Stone JH. Riedel's thyroiditis and multifocal fibrosclerosis are part of the IgG4-related systemic disease spectrum. Arthritis Care Res (Hoboken) 2010;62:1312-8.

51. Deshpande V, Huck A, Ooi E, Stone JH, Faquin WC, Nielsen GP. Fibrosing variant of Hashimoto thyroiditis is an IgG4 related disease. J Clin Pathol 2012;65:725-8.

52. Kawano M, Saeki T. IgG4-related kidney disease--an update. Curr Opin Nephrol Hypertens 2015;24:193-201.

53. Yoshita K, Kawano M, Mizushima I, Hara S, Ito Y, Imai N, et al. Light-microscopic characteristics of IgG4-related tubulointerstitial nephritis: distinction from non-IgG4related tubulointerstitial nephritis. Nephrol Dial Transplant 2012;27:2755-61.

54. Khosroshahi A, Ayalon R, Beck LH Jr, Salant DJ, Bloch DB, Stone JH. IgG4-Related Disease Is Not Associated with Antibody to the Phospholipase A2 Receptor. Int J Rheumatol 2012;2012:139409.

55. Pradhan D, Pattnaik N, Silowash R, Mohanty SK. IgG4-related kidney disease--A review. Pathol Res Pract 2015;211:707-11.

56. Lian L, Wang C, Tian JL. IgG4-related retroperitoneal fibrosis: a newly characterized disease. Int J Rheum Dis 2016;19:1049-55.

57. Tajima M, Nagai R, Hiroi Y. IgG4-related cardiovascular disorders. Int heart J. 2014;55:287-95.

58. Zen Y, Onodera M, Inoue D, Kitao A, Matsui O, Nohara T, et al. Retroperitoneal fibrosis: a clinicopathologic study with respect to immunoglobulin G4. Am J Surg Pathol 2009;33:1833-9.
59. Wick MR, O'Malley DP. Lymphadenopathy associated with IgG4-related disease: Diagnosis \& differential diagnosis. Semin Diag Pathol 2018;35:61-6.

60. Ryu JH, Yi ES. Immunoglobulin G4-Related Disease and the Lung. Clin Chest Med 2016;37:569-78.

61. Zen Y, Kitagawa S, Minato H, Kurumaya H, Katayanagi K, Masuda S, et al. IgG4positive plasma cells in inflammatory pseudotumor (plasma cell granuloma) of the lung. Hum Pathol 2005;36:710-7.

62. Shigemitsu H, Koss MN. IgG4-related interstitial lung disease: a new and evolving concept. Curr Opin Pulm Med 2009;15:513-6.

63. Koizumi S, Kamisawa T, Kuruma S, Tabata T, Chiba K, Iwasaki S, et al Immunoglobulin G4-related gastrointestinal diseases, are they immunoglobulin G4related diseases? World J Gastroenterol 2013;19:5769-74.

64. Obiorah I, Hussain A, Palese C, Azumi N, Benjamin S, Ozdemirli M. IgG4-related disease involving the esophagus: a clinicopathological study. Dis Esophagus 2017;30:1-7.

65. Fujita T, Ando T, Sakakibara M, Hosoda W, Goto H. Refractory gastric ulcer with abundant IgG4-positive plasma cell infiltration: A case report. World J Gastroenterol 2010;16:2183-6.

66. Brito-Zeron P, Ramos-Casals M, Bosch X, Stone JH. The clinical spectrum of IgG4related disease. Autoimmun Rev 2014;13:1203-10.

67. Lu LX, Della-Torre E, Stone JH, Clark SW. Igg4-related hypertrophic pachymeningitis: Clinical features, diagnostic criteria, and treatment. JAMA Neurol 2014;71:785-93.

68. Leporati P, Landek-Salgado MA, Lupi I, Chiovato L, Caturegli P. IgG4-related hypophysitis: a new addition to the hypophysitis spectrum. J Clin Endocrinol Metab 2011;96:1971-80

69. Sato Y, Takeuchi M, Takata K, Ohno K, Iwaki N, Orita Y, et al. Clinicopathologic analysis of IgG4-related skin disease. Mod Pathol 2013;26:523-32.

70. Cheuk W, Chan AC, Lam WL, Chow SM, Crowley P, Lloydd R, et al. IgG4-related sclerosing mastitis: description of a new member of the IgG4-related sclerosing diseases. Am J Surg Pathol 2009;33:1058-64.

71. Bösmüller H, von Weyhern CH, Adam P, Alibegovic V, Mikuz G, Fend F. Paratesticular fibrous pseudotumor--an IgG4-related disorder? Virchows Arch 2011;458:109-13.

72. Kawa S, Skold M, Ramsden DB, Parker A, Harding SJ. Serum IgG4 Concentration in IgG4-Related Disease. Clin Lab 2017;63:1323-37.

73. Culver EL, Sadler R, Simpson D, Cargill T, Makuch M, Bateman AC, et al. Elevated Serum IgG4 Levels in Diagnosis, Treatment Response, Organ Involvement, and Relapse in a Prospective IgG4-Related Disease UK Cohort. Am J Gastroenterol 2016;111:733-43.

74. Wallace ZS, Mattoo H, Carruthers M, Mahajan VS, Della Torre E, Lee H, et al Plasmablasts as a biomarker for IgG4-related disease, independent of serum IgG4 concentrations. Ann Rheum Dis 2015;74:190-5.

75. Khosroshahi A, Wallace ZS, Crowe JL, Akamizu T, Azumi A, Carruthers MN, et al International Consensus Guidance Statement on the Management and Treatment of IgG4-Related Disease. Arth Rheumatol 2015;67:1688-99.

76. Campochiaro C, Ramirez GA, Bozzolo EP, Lanzillotta M, Berti A, Baldissera E, et al. IgG4-related disease in Italy: clinical features and outcomes of a large cohort of patients. Scand J Rheumatol 2016;45:135-45.

77. Hart PA, Topazian MD, Witzig TE, Clain JE, Gleeson FC, Klebig RR, et al. Treatment of relapsing autoimmune pancreatitis with immunomodulators and rituximab: the Mayo Clinic experience. Gut 2013;62:1607-15.

78. Khosroshahi A, Bloch DB, Deshpande V, Stone JH. Rituximab therapy leads to rapid decline of serum IgG4 levels and prompt clinical improvement in IgG4-related systemic disease. Arthritis Rheum 2010;62:1755-62.

79. Carruthers MN, Topazian MD, Khosroshahi A, Witzig TE, Wallace ZS, Hart PA, et al Rituximab for IgG4-related disease: a prospective, open-label trial. Ann Rheum Dis 2015;74:1171-7.

80. Fujita A. Imaging of Sjögren Syndrome and Immunoglobulin G4-Related Disease of the Salivary Glands. Neuroimaging Clin N Am 2018;28:183-97. 